\title{
ENERGY SPECTRA AND FLUXES IN DISSIPATION RANGE OF TURBULENT AND LAMINAR FLOWS
}

\author{
Mahendra K. Verma, ${ }^{1, a)}$ Abhishek Kumar, ${ }^{1, \text { b) }}$ Praveen Kumar, ${ }^{1, \text { c) }}$ Satyajit Barman, ${ }^{1, d)}$ \\ Anando G. Chatterjee, ${ }^{1, e)}$ Ravi Samtaney, ${ }^{2, f)}$ and Rodion Stepanov ${ }^{3, g}$ ) \\ 1) Department of Physics, Indian Institute of Technology, Kanpur 208016, \\ India \\ ${ }^{2)}$ Mechanical Engineering, Division of Physical Sciences and Engineering, \\ King Abdullah University of Science and Technology - Thuwal 23955-6900, \\ Kingdom of Saudi Arabia \\ ${ }^{3)}$ Institute of Continuous Media Mechanics, Korolyov 1, Perm 614013, \\ Russia
}

Two well-known turbulence models that describe the energy spectrum in the inertial and dissipative ranges simultaneously are by Pao (1965) and Pope (2000). In this paper, we compute the energy spectrum $E(k)$ and energy flux $\Pi(k)$ using direct numerical simulations on grids up to $4096^{3}$, and show consistency between the numerical results and the predictions by the aforementioned models. We also construct a model for laminar flows that predicts $E(k) \sim k^{-1} \exp (-k)$ and $\Pi(k) \sim k \exp (-k)$. Our model predictions match with the numerical results. We emphasize differences on the energy transfers in the two flows - they are local in the turbulent flows, and nonlocal in laminar flows.

Keywords: Hydrodynamic turbulence, Turbulence modeling, Direct numerical simulation

\footnotetext{
a)Electronic mail: mkv@iitk.ac.in

b) Electronic mail: abhishek.kir@gmail.com

c) Electronic mail: praveenkumar.hcdu@gmail.com; Present address: BARC, Mumbai

d) Electronic mail: sbarman@iitk.ac.in

e) Electronic mail: anandogc@iitk.ac.in

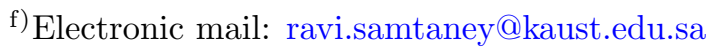

g) Electronic mail: rodion@icmm.ru
} 


\section{INTRODUCTION}

Turbulence is a classic problem with many unresolved issues. The most well-known phenomenology of turbulence is by Kolmogorov ${ }^{1}$, according to which the energy supplied at the large scales cascades to small scales. The wavenumber band dominated by the forcing is called forcing range, while that dominated by dissipation is called dissipative range. The wavenumber band between these two ranges is termed as inertial range. According to Kolmogorov ${ }^{1}$, the energy cascade rate or the spectral energy flux is constant in the inertial range. Quantitatively, the one-dimensional energy spectrum $E(k)$ and the energy flux $\Pi(k)$ in the inertial range are

$$
\begin{aligned}
& E(k)=K_{\mathrm{Ko}} \epsilon^{2 / 3} k^{-5 / 3}, \\
& \Pi(k)=\epsilon,
\end{aligned}
$$

where $\epsilon$ is the energy dissipation rate, and $K_{\text {Ko }}$ is Kolmogorov's constant. The above law has been verified using experiments and high-resolution simulations (see Frisch ${ }^{2}, \mathrm{McComb}^{3}$, Davidson ${ }^{4}$, Ishihara, Gotoh, and Kaneda ${ }^{5}$ and references therein). There is, however, a small correction to the exponent "-5/3" due to intermittency ${ }^{2}$. This issue however is beyond the scope of this paper. Kolmogorov's theory of turbulence and its ramifications have been discussed in detail in several books ${ }^{2-4,6-8}$.

The energy spectrum of Eq. (1) is universal, i.e., it is independent of fluid properties, forcing and dissipative mechanisms, etc. Equation (1) had been extended to the dissipative range in the following manner:

$$
E(k)=K_{\mathrm{Ko}} \epsilon^{2 / 3} k^{-5 / 3} f\left(k / k_{d}\right),
$$

where $f\left(k / k_{d}\right)$ is a universal function, and

$$
k_{d}=\left(\epsilon / \nu^{3}\right)^{1 / 4}
$$

is the dissipation wavenumber scale, also called Kolmogorov's wavenumber. $\mathrm{Pao}^{9}, \mathrm{Pope}^{8}$, and Martínez et al. ${ }^{10}$ modelled $f\left(k / k_{d}\right)$; Pao ${ }^{9}$ proposed that $f(x) \sim \exp \left(-x^{4 / 3}\right)$, but according to Pope $^{8}$

$$
f(x) \sim \exp \left\{-\beta\left(\left[x^{4}+c_{\nu}^{4}\right]^{1 / 4}-c_{\nu}\right)\right\},
$$


where $\beta$ and $c_{\nu}$ are constants. Pope's model ${ }^{8}$ is in good agreement with earlier experimental results (see Saddoogchi and Veeravalli ${ }^{11}$ and references therein). Pao ${ }^{9}$ argued that his predictions fit well with the experimental results of Grant et al. ${ }^{12}$. Martínez et al. ${ }^{10}$ proposed that

$$
E(k) \sim\left(k / k_{d}\right)^{\alpha} \exp \left[-\beta\left(k / k_{d}\right)\right],
$$

and found good agreement between their predictions and numerical results for moderate Reynolds numbers.

There are only a few numerical simulations that have investigated the dissipative spectrum of a turbulent flow. For example, Martínez et al. ${ }^{10}$ computed $E(k)$ for flows with moderate Reynolds number (Re) and showed it to be consistent with the model of Eq. (6). On the other hand, Ishihara et al. ${ }^{13}$ showed that near the dissipation range, Eq. (6) is a good approximation also for high Reynolds number flows. The energy flux in the dissipative regime of a turbulent flow has not been investigated in detail, either by numerical simulation or experiments. Note that $\Pi(k)$ in the dissipative range is assumed to be small and rapidly decreasing, and it is typically ignored. In the present paper, we perform turbulence simulations on very high-resolution grids (up to $4096^{3}$ ), and compare the numerically-computed $E(k)$ and $\Pi(k)$ with those predicted by various models.

Laminar flows are ubiquitous in nature; some examples of such flows are-blood flow in arteries, micro and nano fluidics ${ }^{14}$, mantle convection inside the $\operatorname{Earth}^{15}$, laminar dynamo, and passive scalar turbulence with large Schmidt number ${ }^{16}$. Hence, models of laminar flows are very useful. In this paper, we construct a spectral model for such flows. We consider laminar flows with Reynolds number of the order of unity. For such flows, the nonlinearity and the energy flux are quite small. Researchers have studied the kinetic energy spectrum for such flows and predicted this to be of the form $\exp \left(-k^{2}\right)$ or $\exp (-k)^{10,17-20}$. For example, see Eq. (6) proposed by ${ }^{10}$. In the present paper, we show that $E(k) \sim k^{-1} \exp \left(-k / \bar{k}_{d}\right)$ and $\Pi(k) \sim k \exp \left(-k / \bar{k}_{d}\right)$, where $\bar{k}_{d}$ is a measure of dissipation wavenumber, describe the energy spectrum and flux of laminar flows. We also performed direct numerical simulations (DNS) for Re ranging from 17.6 to 49 and verified our model predictions with numerical data.

According to Kolmogorov's theory of turbulence, the energy transfers among the inertialrange wavenumber shells are forward and local ${ }^{21-23}$. That is, a wavenumber shell (say $m$ ) transfers maximal energy to its nearest forward neighbour shell $(m+1)$, and receives maximal energy from its previous neighbour shell $(m-1)$. The aforementioned phenomena 
have been verified using numerical simulations. However, there has not been a definitive shell-to-shell energy transfer computation for the dissipative regime of a turbulent flow. Similar computation for laminar flows is also lacking. In this paper, we show that the shellto-shell energy transfers in the dissipative regime of a turbulent flow are local. However, these transfers for laminar flows are nonlocal.

There are a number of analytical works for modeling turbulence, namely quasi-normal approximation, eddy-damped quasi-normal Markovian ${ }^{24}$, direct-interaction approximation ${ }^{25}$, etc. These sophisticated models attempt to compute higher-order correlations and related quantities using various closure schemes ${ }^{2,6}$. The focus of the present paper is on the validation of some of the popular spectral models that predict the energy spectrum and fluxes. We remark that such studies are very important for applications encountered by engineers, and geo-, astro-, and atmospheric physicists.

The outline of the paper is as follows: In Sec. II, we describe the turbulence models of Pao and Pope, as well as our model for laminar flows. Sec. III contains numerical results of high-resolution simulations of turbulent and laminar flows. We conclude in Sec. IV.

\section{MODEL DESCRIPTION}

An incompressible fluid flow is described by the Navier-Stokes equations:

$$
\begin{aligned}
\frac{\partial \mathbf{u}}{\partial t}+(\mathbf{u} \cdot \nabla) \mathbf{u} & =-\frac{1}{\rho} \nabla p+\nu \nabla^{2} \mathbf{u}+\mathbf{f} \\
\nabla \cdot \mathbf{u} & =0
\end{aligned}
$$

where $\mathbf{u}$ is the velocity field, $p$ is the pressure field, $\nu$ is the kinematic viscosity, and $\mathbf{f}$ is the force field. We take the density $\rho$ to be a constant, equal to unity. In Fourier space the above equations are transformed to (e.g. see Lesieur $^{7}$ )

$$
\begin{aligned}
\left(\frac{\partial}{\partial t}+\nu k^{2}\right) \mathbf{u}(\mathbf{k}, t) & =-i \mathbf{k} p(\mathbf{k}, t)-i \sum_{\mathbf{k}=\mathbf{p}+\mathbf{q}} \mathbf{k} \cdot \mathbf{u}(\mathbf{q}) \mathbf{u}(\mathbf{p})+\mathbf{f}(\mathbf{k}), \\
\mathbf{k} \cdot \mathbf{u}(\mathbf{k}) & =0
\end{aligned}
$$

where $\mathbf{u}(\mathbf{k}), p(\mathbf{k})$, and $\mathbf{f}(\mathbf{k})$ are the Fourier transforms of $\mathbf{u}, p$, and $\mathbf{f}$ respectively. The above equations yield the following equation for one-dimensional energy spectrum $E(k, t)^{7}$ :

$$
\frac{\partial E(k, t)}{\partial t}=T(k, t)-2 \nu k^{2} E(k, t)+\mathcal{F}(k, t)
$$


where $T(k, t)$ is the energy transfer to the wavenumber shell $k$ due to nonlinearity, $\mathcal{F}(k, t)$ is the energy feed by the force, and $-2 \nu k^{2} E(k, t)$ is the dissipation spectrum. Note that $T(k, t)=-\partial \Pi(k, t) / \partial k$. In Kolmogorov's model, the energy supply rate $\mathcal{F}(k, t)$ is active at large length scales (for $k \approx k_{f}$, where $k_{f}$ is the forcing wavenumber), and it is absent in the inertial and dissipative range. In a statistically steady state, $\partial E(k, t) / \partial t=0$. Hence the energy flux $\Pi(k)$ varies with $k$ as

$$
\frac{d}{d k} \Pi(k)=-2 \nu k^{2} E(k) .
$$

The turbulence model of $\mathrm{Pao}^{9}$ is based on the above equation.

In hydrodynamics, it is convenient to work with dimensionless quantities. We nondimensionalize $k$ using the Kolmogorov wavenumber $k_{d}$, which is defined by (4). The energy flux $\Pi(k)$ is nondimensionalized using $\epsilon$. Hence,

$$
\begin{aligned}
\tilde{k} & =\frac{k}{k_{d}}, \\
\tilde{\Pi}(\tilde{k}) & =\frac{\Pi(k)}{\epsilon} .
\end{aligned}
$$

We compensate Eq. (3) such that

$$
\tilde{E}(\tilde{k})=\frac{E(k)}{K_{\mathrm{Ko}} \epsilon^{2 / 3} k^{-5 / 3}}=f_{\nu}(\tilde{k}) .
$$

Substitution of the above variables in Eq. (12) yields

$$
\frac{d}{d \tilde{k}} \tilde{\Pi}(\tilde{k})=-2 K_{\mathrm{Ko}} \tilde{k}^{1 / 3} f_{\nu}(\tilde{k}) .
$$

After the above introduction, we describe some of the important spectral models for turbulent and laminar flows.

\section{A. Pao's model of turbulent flow}

In this paper, we discuss the models of $\mathrm{Pao}^{9}$ and $\mathrm{Pope}^{8}$. To contrast the two models, we label the energy fluxes and spectra of these models differently. For Pao's model, we label the energy spectrum, flux, nondimensional dissipative function as $\tilde{E}^{(1)}(\tilde{k}), \tilde{\Pi}^{(1)}(\tilde{k})$ and $f_{\nu}^{(1)}(\tilde{k})$ respectively; for Pope's model ${ }^{8}$ we use $\tilde{E}^{(2)}(\tilde{k}), \tilde{\Pi}^{(2)}(\tilde{k})$ and $f_{\nu}^{(2)}(\tilde{k})$ for the corresponding quantities. 
In Eq. $(16), \tilde{\Pi}(\tilde{k})$ and $f_{\nu}(\tilde{k})$ are two unknown functions. Hence, to close the problem, $\mathrm{Pao}^{9}$ assumed that the ratio $\Pi(k) / E(k)$ is independent of $\nu$. Using dimensional analysis, the ratio can be expressed using $\epsilon$ and $k$ as

$$
\frac{\Pi(k)}{E(k)}=\alpha^{-1} \epsilon^{1 / 3} k^{5 / 3},
$$

where coefficient $\alpha$ is chosen as $K_{\mathrm{Ko}}$. In terms of the nondimensional variables,

$$
\tilde{\Pi}^{(1)}(\tilde{k})=\tilde{E}^{(1)}(\tilde{k})=f_{\nu}^{(1)}(\tilde{k})
$$

In other words, the dissipative functions for both $\tilde{E}(k)$ and $\tilde{\Pi}(k)$ should be of the same form. Thus Eq. (16) yields

$$
f_{\nu}^{(1)}(\tilde{k})=\exp \left(-\frac{3}{2} K_{\mathrm{Ko}} \tilde{k}^{4 / 3}\right)
$$

\section{B. Pope's model of turbulent flow}

Pope $^{8}$ constructed another popular model for the turbulent flow. For this model, we denote the energy spectrum, flux, nondimensional dissipative function $f_{\nu}(\tilde{k})$ as $\tilde{E}^{(2)}(\tilde{k})$, $\tilde{\Pi}^{(2)}(\tilde{k})$ and $f_{\nu}^{(2)}(\tilde{k})$ respectively. Pope ${ }^{8}$ proposed that

$$
E^{(2)}(k)=K_{\mathrm{Ko}} \epsilon^{2 / 3} k^{-5 / 3} f_{L}(k L) f_{\nu}^{(2)}\left(k / k_{d}\right)
$$

with the functions $f_{L}(k L)$ and $f_{\nu}\left(k / k_{d}\right)$ specifying the large-scale and dissipative-scale deviations from "-5/3" power law, respectively:

$$
\begin{aligned}
& f_{L}(k L)=\left(\frac{k L}{\left[(k L)^{2}+c_{L}\right]^{1 / 2}}\right)^{5 / 3+p_{0}}, \\
& f_{\nu}^{(2)}(\tilde{k})=\exp \left[-\beta\left\{\left[\tilde{k}^{4}+c_{\nu}^{4}\right]^{1 / 4}-c_{\nu}\right\}\right],
\end{aligned}
$$

where the $c_{L}, c_{\nu}, p_{0}, \beta$ are constants. Since we focus on the inertial and dissipative ranges, for which $k \gg 1 / L$, we set $f_{L}(k L)=1$. In the high Reynolds number limit, $c_{\nu} \approx 0.47 \beta^{1 / 3} / K_{\mathrm{Ko}}{ }^{8}$. We choose $\beta=5.2$ as prescribed by $\operatorname{Pope}^{8}$. Substitution of $\tilde{E}^{(2)}(\tilde{k})$ and $\tilde{\Pi}^{(2)}(\tilde{k})$ in Eq. (16) yields the following solution

$$
\tilde{\Pi}^{(2)}(\tilde{k})=\tilde{\Pi}^{(2)}\left(\tilde{k}_{0}\right)-2 K_{\text {Ko }} \int_{\tilde{k}_{o}}^{\tilde{k}} \tilde{k}^{\prime 1 / 3} f_{\nu}^{(2)}\left(\tilde{k}^{\prime}\right) d \tilde{k}^{\prime},
$$

which is solved numerically with $f_{\nu}^{(2)}(\tilde{k})$ of Eq. $(22)$. We set $\tilde{\Pi}^{(2)}\left(\tilde{k}_{0}\right)=1$ at small $k_{0}$. Refer to $\mathrm{Pope}^{8}$ for the detailed derivation of above relation. 


\section{Model for the laminar flows}

We will show later that the models of $\mathrm{Pao}^{9}$ and $\mathrm{Pope}^{8}$ do not provide satisfactory description of laminar flows with $\operatorname{Re} \approx O(1)$. Earlier Martínez et al. ${ }^{10}$ had proposed that the energy spectrum in the dissipative regime has the following empirical form:

$$
E(k) \sim\left(k / k_{d}\right)^{\alpha} \exp \left[-\beta\left(k / k_{d}\right)\right]
$$

where $\alpha$ and $\beta$ are constants. Based on the above form, we propose a new model for the energy spectrum of laminar flows, according to which

$$
E(k)=u_{\mathrm{rms}}^{2} k^{-1} f_{L}\left(k / \bar{k}_{d}\right) \exp \left(-k / \bar{k}_{d}\right),
$$

where $u_{\mathrm{rms}}$ is the rms velocity of the flow, and

$$
\bar{k}_{d}=\frac{\sqrt{\mathrm{Re}}}{1.3 L}
$$

with $L$ as the box size, and $\operatorname{Re}=u_{\text {rms }} L / \nu$ is the Reynolds number. Note that $\bar{k}_{d}$ of a laminar flow differs from $k_{d}$, the Kolmogorov's wavenumber defined in Eq. (4). Interestingly, the above form of $\bar{k}_{d}$ follows from

$$
\tilde{k}_{d}=\left(\frac{\epsilon}{\nu^{3}}\right)^{1 / 4} \sim\left(\frac{\nu U^{2}}{L^{2} \nu^{3}}\right)^{1 / 4} \sim \frac{\sqrt{\mathrm{Re}}}{L} .
$$

Substitution of Eq. (25) in the expression for the energy dissipation rate yields

$$
\begin{aligned}
\epsilon & =\int_{0}^{\infty} 2 \nu k^{2} E(k) d k \\
& =2 \nu u_{\mathrm{rms}}^{2} \bar{k}_{d}^{2} \int_{0}^{\infty} \bar{k} f_{L}(\bar{k}) \exp (-\bar{k}) d \bar{k} \\
& =2 \nu u_{\mathrm{rms}}^{2} \bar{k}_{d}^{2} A
\end{aligned}
$$

where $\bar{k}=k / \bar{k}_{d}$, and $A=\int_{0}^{\infty} \bar{k} f_{L}(\bar{k}) \exp (-\bar{k}) d \bar{k}$ is a nondimensional constant. From numerical simulation we observe that $A \sim f_{L}(\bar{k}) \sim \mathrm{Re}^{-3}$. Hence, for the laminar regime, we nondimensionalize energy spectrum and energy flux as follows:

$$
\begin{aligned}
\bar{E}(\bar{k}) & =\frac{E(k) k \mathrm{Re}^{3}}{u_{\mathrm{rms}}^{2}}, \\
\bar{\Pi}(\bar{k}) & =\frac{\Pi(k)}{\epsilon}=\frac{\Pi(k)}{2 \nu A u_{\mathrm{rms}}^{2} \bar{k}_{d}^{2}} .
\end{aligned}
$$


Substitution of Eq. (25) in Eq. (12) yields

$$
\frac{d \bar{\Pi}(\bar{k})}{d \bar{k}}=-\frac{\bar{k}}{A} f_{L}(\bar{k}) \exp (-\bar{k})
$$

whose solution is

$$
\bar{\Pi}(\bar{k})=\bar{\Pi}\left(\bar{k}_{0}\right)-\frac{1}{A} \int_{\overline{k_{0}}}^{\bar{k}} \bar{k} f_{L}(\bar{k}) \exp (-\bar{k}) d \bar{k},
$$

where $\bar{k}_{0}$ is the reference wavenumber. Note that for $k>k_{f}$, the dimensionless energy spectrum and flux are of the following form:

$$
\begin{aligned}
\bar{E}(\bar{k}) & =\exp (-\bar{k}), \\
\bar{\Pi}(\bar{k}) & =(1+\bar{k}) \exp (-\bar{k}) .
\end{aligned}
$$

In the next section we verify the above models using numerical simulations.

\section{NUMERICAL VALIDATION OF THE MODELS}

We perform direct numerical simulation of incompressible Navier-Stokes equation [see Eqs. $(7,8)]$ in turbulent and laminar regimes, and compute the energy spectra and fluxes

for various cases. We employ pseudo-spectral code Tarang ${ }^{26}$ for our simulations. We use the fourth-order Runge-Kutta scheme for time advancement with variable $\Delta t$, which is chosen using the CFL condition. The pseudo-spectral method produces aliasing error, which is overcome by setting $(1 / 3)$ rd of the Fourier modes to zero. This dealiasing procedure is referred to as " $2 / 3 "$ rule $^{27}$.

We compute the energy spectra and fluxes for all the numerical runs during the steady state. The energy spectrum $E(k)$ is computed using ${ }^{28}$

$$
E(k)=\frac{4 \pi}{M} \sum_{k-1<k^{\prime} \leq k} \frac{1}{2}\left|\mathbf{u}\left(\mathbf{k}^{\prime}\right)\right|^{2}\left|\mathbf{k}^{\prime}\right|^{2}
$$

where $M$ is the number of modes in the shell between wavenumbers $k-1$ and $k$. Note that the above formula reduces bias in the energy spectrum at low wavenumbers ${ }^{28}$. The energy flux $\Pi\left(k_{0}\right)$, the rate of kinetic energy emanating from the wavenumber sphere of radius $k_{0}$, is computed using the following formula ${ }^{29,30}$ :

$$
\Pi\left(k_{0}\right)=\sum_{k>k_{0}} \sum_{p \leq k_{0}} \operatorname{Im}\left\{[\mathbf{k} \cdot \mathbf{u}(\mathbf{k}-\mathbf{p})]\left[\mathbf{u}^{*}(\mathbf{k}) \cdot \mathbf{u}(\mathbf{p})\right]\right\}
$$




\section{A. Turbulent Flow}

We perform our turbulence simulations on $512^{3}, 1024^{3}$, and $4096^{3}$ grids. We employ periodic boundary conditions on all sides of a cubic box of size $(2 \pi)^{3}$. To obtain a steady turbulent flow, we apply random forcing ${ }^{31}$ in the wavenumber band $2 \leq k \leq 4$ for $1024^{3}$ and $4096^{3}$ grids, but in the band $1 \leq k \leq 3$ for $512^{3}$ grid. We choose a random initial condition for the $512^{3}$-grid simulation. The steady-state data of $512^{3}$ was used as an initial condition for the $1024^{3}$-grid run, whose steady-state data is used for $4096^{3}$-grid simulation. In all the three cases, the velocity field at the small scales are well resolved because $k_{\max } \eta$ is always greater than 1.5, where $k_{\max }$ is the highest wavenumber represented by the grid points, and $\eta \sim 1 / k_{d}$ is the Kolmogorov's length. The Reynolds numbers for the $512^{3}, 1024^{3}$, and $4096^{3}$ grid simulations are $5.7 \times 10^{3}, 1.4 \times 10^{4}$, and $6.8 \times 10^{4}$ respectively. We observe that the energy flux in the inertial range, the energy dissipation rate, and the energy supply rate by the forcing are equal to each other within 2-4\%. The energy supply rate is chosen as 0.1 , but the energy dissipation rate, as well as the energy flux, vary from 0.096 to 0.102. The parameters of our runs for turbulent flows are listed in Table I. In the Table, we report the value of $\epsilon /\left(u_{\mathrm{rms}}^{3} / L\right)$ which is approximately unity for all three simulations.

TABLE I. Parameters of our direct numerical simulations (DNS) for turbulent flow: grid resolution; kinematic viscosity $\nu$, Reynolds number Re, Kolmogorov constant $K_{\text {Ko }}$, Kolmogorov wavenumber $k_{d}, k_{\max } \eta$, and $\epsilon /\left(u_{\mathrm{rms}}^{3} / L\right)$.

\begin{tabular}{ccccccc}
\hline \hline Grid & $\nu$ & Re & $K_{\text {Ko }}$ & $k_{d}$ & $k_{\max } \eta$ & $\epsilon /\left(u_{\mathrm{rms}}^{3} / L\right)$ \\
\hline $512^{3}$ & $10^{-3}$ & $5.7 \times 10^{3}$ & $2.2 \pm 0.2$ & $9.8 \times 10^{1}$ & 2.5 & 0.9 \\
$1024^{3}$ & $4 \times 10^{-4}$ & $1.4 \times 10^{4}$ & $1.85 \pm 0.05$ & $2.1 \times 10^{2}$ & 2.4 & 1.0 \\
$4096^{3}$ & $8 \times 10^{-5}$ & $6.8 \times 10^{4}$ & $1.75 \pm 0.05$ & $6.6 \times 10^{2}$ & 3.1 & 1.0 \\
\hline
\end{tabular}

Figure 1(a, b, c) exhibits the normalized spectra $\tilde{E}(\tilde{k})$ for the $512^{3}, 1024^{3}$, and $4096^{3}$ grid simulations. Note that the grey regions in the figures denote the forcing band. The plots show that the numerical $\tilde{E}(\tilde{k})$ are close to the predictions of both Pao's and Pope's models. We also compute the Kolmogorov's constant $K_{\text {Ko }}$ using

$$
K_{\mathrm{Ko}}=\epsilon^{-2 / 3}\left\langle E(k) k^{5 / 3}\right\rangle,
$$


where an average is taken over scales in the inertial range just after forcing scale. As shown in Table I, the values of $K_{\text {Ko }}$ varies from 1.75 to 2.2 with errors in the range of $3 \%$ to $9 \%$. These values are in the same range as those reported earlier ${ }^{32-37}$. The numerical estimates of $K_{\text {Ko }}$ from DNS appears to be slightly larger than its theoretical value, which is approximately $1.6^{38,39}$. The increases in the value of $K_{\mathrm{Ko}}$ in DNS is possibly due to the fact that the inertial range is not completely established, and the the inertial range $E(k)$ is affected by the forcing and dissipation scales even for $4096^{3}$ grid simulations. Similar enhancement in the value of

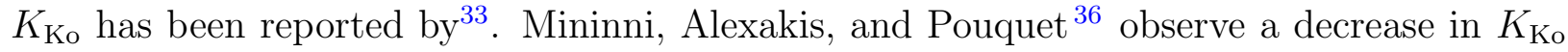
with the Reynolds number, as in our simulations.

An examination of the normalized spectrum $\tilde{E}(\tilde{k})$ indicates a bump near the transition region between the inertial range and dissipation range $(0.04 \lesssim \tilde{k} \lesssim 0.2)$, which is due to the bottleneck effect ${ }^{11,33,37,40-43}$. The predicted $\tilde{E}(\tilde{k})$ by the models of Pao and Pope gradually decrease with $\tilde{k}$. Thus, these models do not capture the bottleneck effect. This is possibly because Pao's and Pope's models do not address the fluctuations in the energy flux. Nevertheless, the spectrum in the dissipative range is captured reasonably well by these models.

In Fig. 1(d, e, f) we plot the nondimensionalized energy fluxes $\tilde{\Pi}(\tilde{k})$ computed using the DNS data. We observe that $\tilde{\Pi}(\tilde{k})$ are approximately constant in the inertial range, consistent with Kolmogorov's theory ${ }^{1}$. In the same plot, we present the energy fluxes computed using the Pao's and Pope's models (Eqs. (19) and (23)). In the inertial range, the predictions of both the models are in good agreement with the DNS results. In the dissipation range, the predictions of Pao's model are close to the numerical values of $\tilde{\Pi}(\tilde{k})$ for $\operatorname{Re}=5.7 \times 10^{3}$ and Re $=4 \times 10^{-4}$, but Pao's prediction for $\mathrm{Re}=8 \times 10^{-5}$ is slightly larger than the numerical values. The predictions of Pope's model are systematically lower than their corresponding numerical counterparts. The suppression of the energy flux at the bottleneck region may be a reason for the discrepancy between the predictions of Pao's model and the numerical values. The increase in $E(k)$ at the bottleneck region leads to an enhanced viscous dissipation, and thus a lower energy flux. As expected, this feature get more pronounced at larger Reynolds numbers.

In addition, we also study the properties of the shell-to-shell energy transfers for the numerical data of $4096^{3}$ grid. For this purpose we divide the Fourier space into 40 shells, whose centers are at the origin $\mathbf{k}=(0,0,0)$. The inner and outer radii of the shells are 

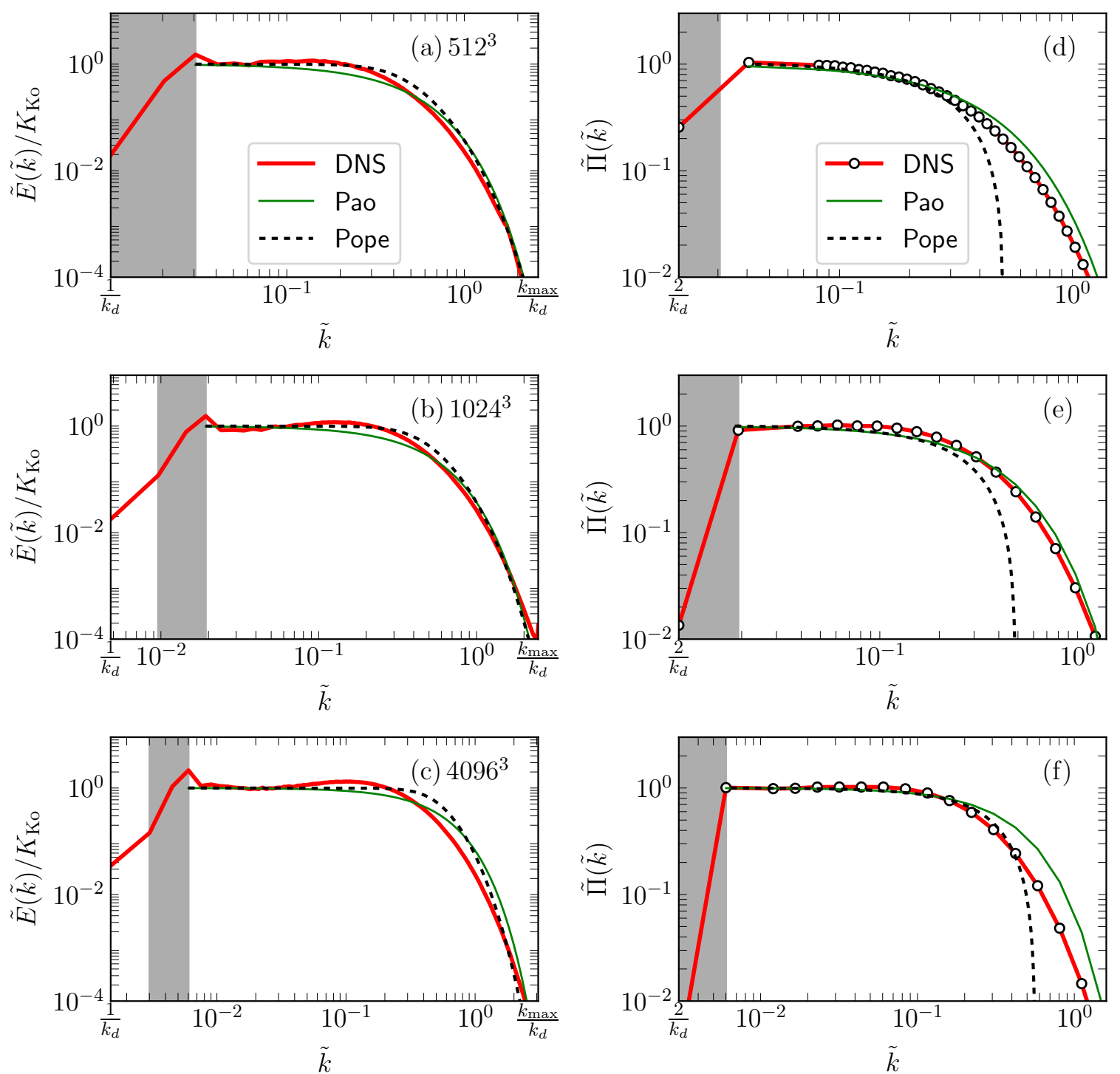

FIG. 1. For the grid resolutions of $512^{3}, 1024^{3}$, and $4096^{3}$ : (a,b,c) Plots of the normalized energy spectrum $\tilde{E}(\tilde{k})$ vs. $\tilde{k} ;(\mathrm{d}, \mathrm{e}, \mathrm{f})$ plots of normalized energy flux $\tilde{\Pi}(\tilde{k})$ vs. $\tilde{k}$. See Eqs. $(14,15)$ for definitions. The plots include the spectra and fluxes computed using numerical data (thick solid line), and the model predictions by Pao (thin solid line) and Pope (dashed line). The grey regions indicate the forcing range.

$k_{n-1}$ and $k_{n}$ respectively, where $k_{n}=\left\{0,2,4,8 \times 2^{s(n-3)}, \ldots, 2048\right\}$ with $s=1 / 5$. The shells are logarithmically binned ${ }^{44}$. Note that the 27 th shell, whose wavenumber range is $194 \leq k \leq 223$, separates the dissipative range from the inertial range. In Fig. 2(a), we exhibit the shell-to-shell energy transfers for the whole range, while Fig. 2(b) shows these 

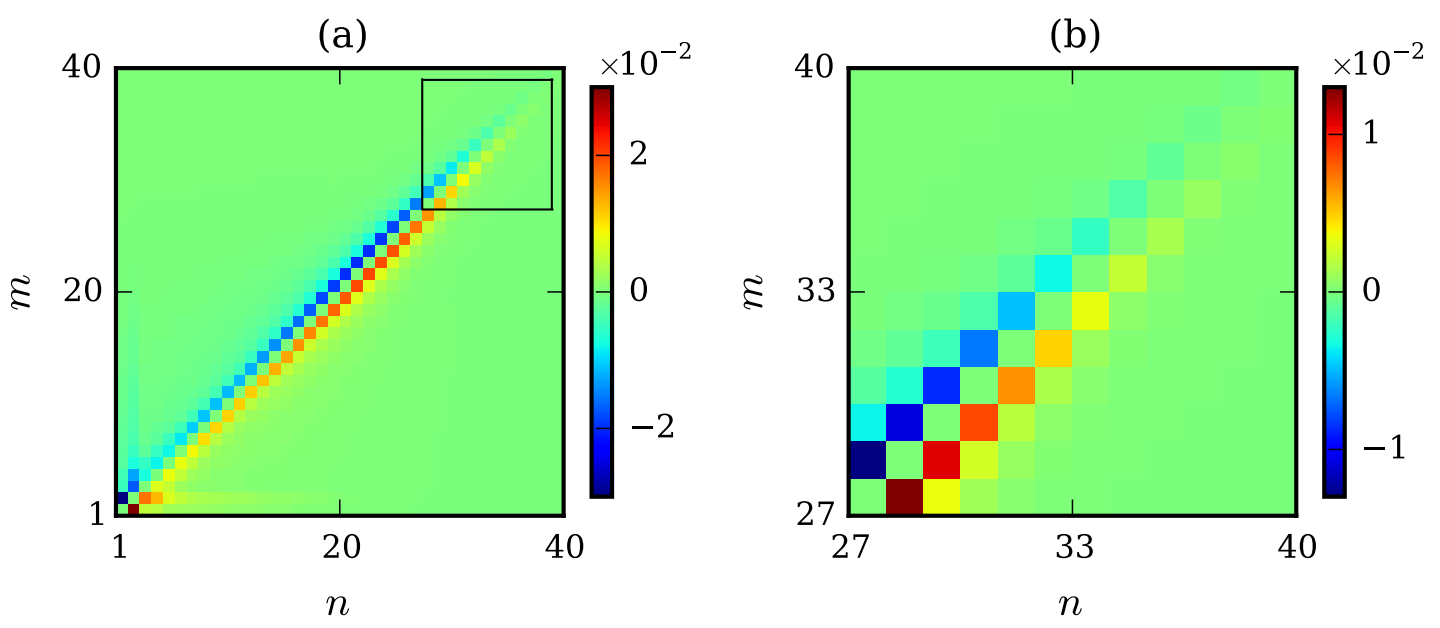

FIG. 2. For the turbulent simulation on $4096^{3}$ grid: Plots of the shell-to-shell energy transfer rates (a) for the whole wavenumber range, (b) for the dissipative range corresponding to the boxed region of subfigure (a). Here $m$ denotes the giver shell, while $n$ denotes the receiver shell. Our results indicate forward and local energy transfers in the inertial as well as in the dissipative wavenumber range.

transfers for the dissipative range only. As expected, in the inertial range, shell $m$ transfers energy dominantly to shell $m+1$, and it receives energy from shell $m-1$. Hence, the shellto-shell energy transfers are forward and local ${ }^{21-23}$. Interestingly, similar behaviour, forward and local energy transfer, is also observed for the wavenumber shells in the dissipative regime. This is essentially because the correlations induced by forcing at small wavenumbers are lost deep inside the inertial and dissipative ranges.

\section{B. Laminar Flow}

We performed direct numerical simulation of laminar flows on $64^{3}$ grid for four sets of parameters of Table II. We choose random initial condition for all our simulations. To reach a steady state, we employ random forcing in the wavenumber band $2 \leq k \leq 4$ with a energy supply rate of unity. The Reynolds numbers of these simulations range from 17.6 to 49. For the steady state, the energy dissipation rate ranges from 0.999 to 1.002 , and which is within $0.1-0.2 \%$ of the energy supply rate. 
TABLE II. Parameters of our direct numerical simulations (DNS) for laminar flows: kinematic viscosity $\nu$; Reynolds number Re; Kolmogorov's wavenumber $\bar{k}_{d}$; and $k_{\max } \eta$.

\begin{tabular}{cccc}
\hline \hline$\nu$ & $\operatorname{Re}$ & $\bar{k}_{d}$ & $k_{\text {max }} \eta$ \\
\hline 0.12 & 49 & 0.9 & 6.52 \\
0.16 & 32.4 & 0.7 & 8.09 \\
0.20 & 23.1 & 0.6 & 9.57 \\
0.24 & 17.6 & 0.5 & 10.97 \\
\hline
\end{tabular}
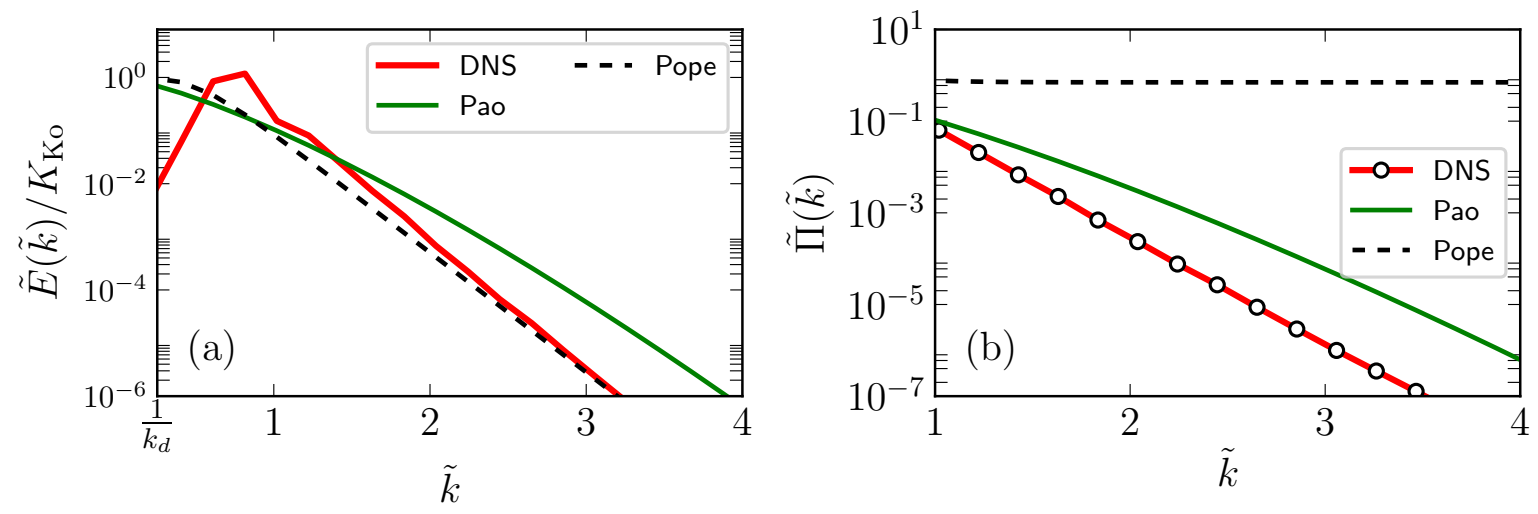

FIG. 3. For the laminar flow simulation with $R e=49$ : Plots of (a) the normalized energy spectrum $\tilde{E}(\tilde{k})$; (b) the normalized energy flux $\tilde{\Pi}(\tilde{k})$. See Eqs. $(14,15)$ for definitions. The model predictions of Pao (thin line) and Pope (dashed line) do not match with the numerical plots.

We attempt to verify whether Pope's and/or Pao's models describe the energy spectrum and flux of laminar flows. Towards this goal, for the laminar flow with Re =49, in Fig. 3(a,b) we plot the normalized energy spectrum $\tilde{E}(\tilde{k})$ and the normalized energy flux $\tilde{\Pi}(\tilde{k})$. In the figure, we also plot the predictions of Pao's and Pope's models. These predictions differ significantly from the numerical results. Thus, Pao's and Pope's models do not describe $E(k)$ and $\Pi(k)$ of the laminar flows. We will show below that the model discussed in Sec. II C describes the numerical results quite well.

In Fig. 4, we plot $\bar{E}(\bar{k})=\operatorname{Re}^{3} E(k) k / u_{\text {rms }}^{2}$ (see Eq. (33)) computed using the numerical data for $\operatorname{Re}=49,32.4,23.1$ and 17.6. We find that for $k>k_{f}$, all $\bar{E}(\bar{k})$ 's merge into a single 


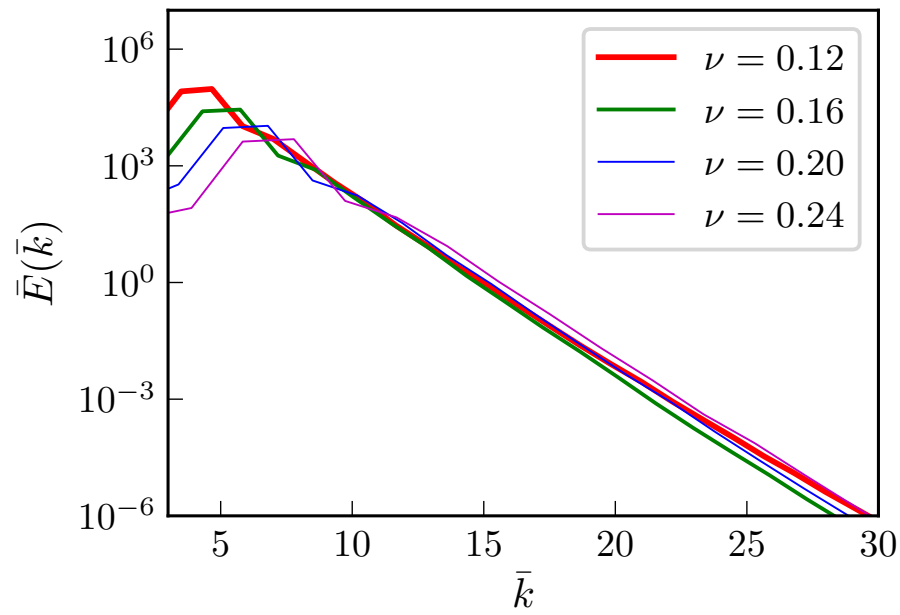

FIG. 4. For the laminar flow simulations, plots of the normalized energy spectra of $\tilde{E}(\tilde{k})$ of Eq. (29). All the plots merge into a single curve for $k>k_{f}$.

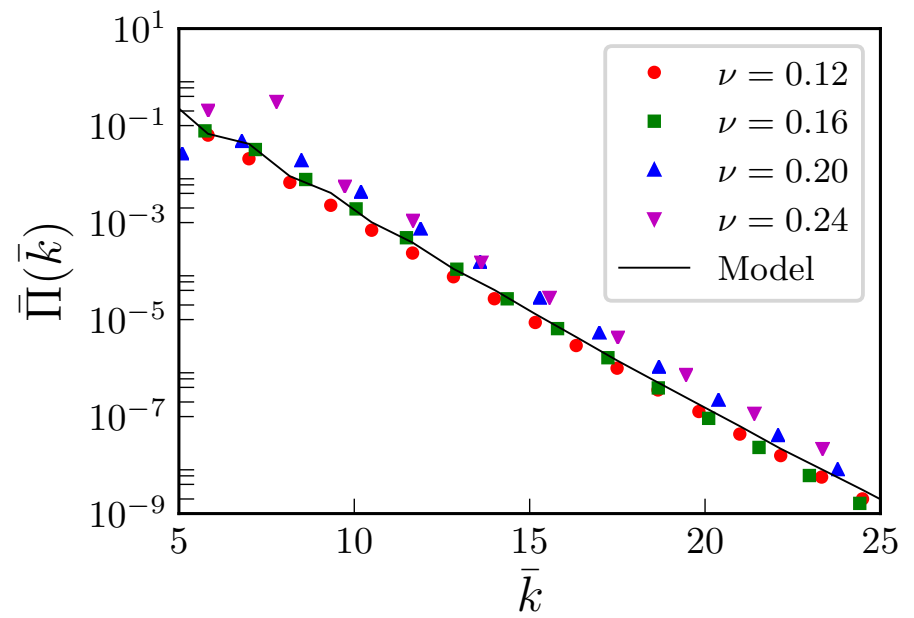

FIG. 5. For the laminar flow simulations, plots of the normalized energy fluxes. All the plots merge into a single curve.

curve indicating that $\bar{E}(\bar{k})$ is a universal function in this range. Also, $\bar{E}(\bar{k}) \sim \exp (-\bar{k}) / k$ verifying the model predictions (see Sec. II C). Note that the $\bar{E}(\bar{k})$ for low $k$ does not merge into a single curve.

In Fig. 5, we plot the normalized energy flux $\bar{\Pi}(\bar{k})$ for the four simulations. We observe that the function $k \exp (-\bar{k})$ provides a good fit to the numerical $\bar{\Pi}(\bar{k})$, consistent with the 
model predictions. The aforementioned consistency between the numerical results and model predictions yields strong credence to the model.

We compute the shell-to-shell energy transfers using the numerical data for $\operatorname{Re}=49$ and 17.6. We divide the Fourier space into 32 shells, whose centers are at the origin $\mathbf{k}=(0,0,0)$. The inner and outer radii of the shells are $k_{n-1}$ and $k_{n}$ respectively, where $k_{n}=\{0,2,4,8,8 \times$ $\left.2^{s(n-3)}, \ldots, 32\right\}$ with $s=1 / 27$. The forcing wavenumber band $2 \leq k \leq 4$ is inside the 2 nd shell. In Fig. 6(a,b), we exhibit the shell-to-shell energy transfers for $\operatorname{Re}=49$ and 17.6 respectively. We observe that the most dominant energy transfers are from the forcing band to the shells of larger radii, e.g., from shell 2 to shells $3-10$ for $\operatorname{Re}=49$, and to the shells $3-7$ for $\operatorname{Re}=17.6$. Thus, the energy transfers for laminar flows are nonlocal. This is because in laminar flows, the velocity field appears to be correlated with the forcing field. This issue needs further investigation.
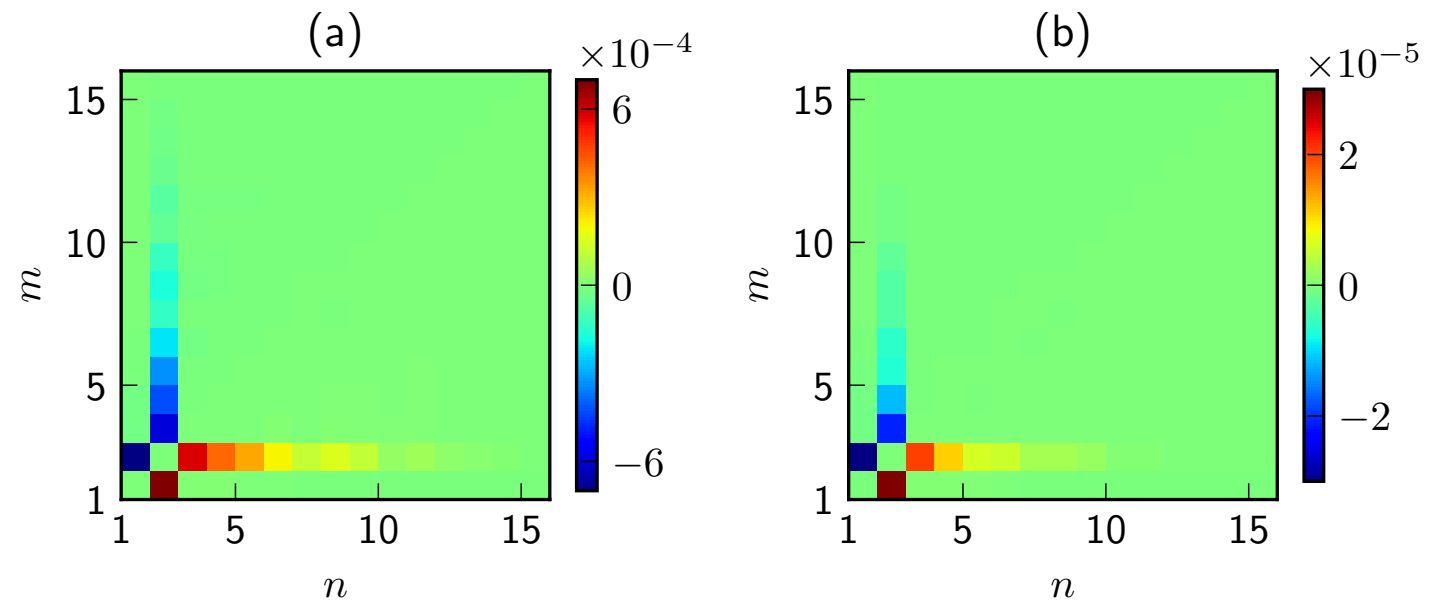

FIG. 6. Plots of the shell-to-shell energy transfer rates for the laminar simulation with (a) $\operatorname{Re}=49$, (b) $\operatorname{Re}=17.6$. Here $m$ denotes the giver shell, while $n$ denotes the receiver shell. The forcing wavenumbers belong to the 2 nd shell.

\section{CONCLUSIONS}

Turbulence is a complex problem, hence we rely on turbulence models. $\mathrm{Pao}^{9}$ and $\mathrm{Pope}^{8}$ constructed turbulence models that explains the turbulence behaviour in the inertial and 
dissipative ranges. Several experimental results on the energy spectrum have been compared with the model predictions, and they match with each other quite well. To best of our knowledge, ours is the first numerical verification of Pao's and Pope's models ${ }^{8,9}$. The present paper shows that the predictions of the above models and numerical results are consistent with each other, except minor differences in the energy flux in the dissipation regime.

The aforementioned models for turbulent flows, however, have certain deficiencies. The hump in the energy spectrum near the beginning of dissipation range is related to the bottleneck effect ${ }^{37,43,45}$; this hump is not captured by of Pao's and Pope's models. Also, the numerical values of the energy flux in the dissipative regime differ from the model predictions by a small amount. Thus, the models of $\mathrm{Pao}^{9}$ and $\mathrm{Pope}^{8}$ need to be revised. It is also interesting to note that Pao's model ${ }^{9}$ does not involve any free parameter (except Kolmogorov's constant $\left.K_{\mathrm{Ko}}\right)$ in comparison to several free parameters in Pope's model. The parameters of Pope's model are chosen so as to fit with $E(k)$ derived from experiments. We show that for turbulent flows, the shell-to-shell energy transfers are forward and local in both inertial and dissipative ranges.

In this paper, we also present a new model for the energy spectrum and flux of laminar flows with Re $\sim 1$. According to our model, the energy spectrum and flux exhibit exponential behaviour $(\exp (-k))$. We verify the model predictions using numerical simulations. For the laminar flows, we also show that the energy transfers are nonlocal and forward; the forcing wavenumbers supply energy to different shells. For moderate Re $(\sim 25)$ to large Re, Martínez et $a{ }^{10}{ }^{10}$ argued that the energy spectrum is of the form Eq. (24), where the parameters $\alpha$ and $\beta$ depend on the Reynolds numbers and length scales. Our model for the laminar flow is simpler and more suitable than that by Martínez et al. ${ }^{10}$.

It is important to differentiate the behaviour of laminar flows with that of highly viscous flows for which $\operatorname{Re} \rightarrow 0$. For the highly viscous flows, the nonlinear term vanishes, and the velocity field is computed using $\nu \nabla^{2} \mathbf{u}=-\mathbf{f}$, or $\mathbf{u}(\mathbf{k})=\mathbf{f}(\mathbf{k}) /\left(\nu k^{2}\right)$ in Fourier space. In such flows, the energy flux vanishes due to the absence of the nonlinear term. An injection of weak nonlinearity in a highly viscous flow will induce a small flux that can be computed perturbatively.

In summary, in this paper, we verify the predictions of Pao's and Pope's models ${ }^{8,9}$ for turbulent flows. We also show that the energy spectrum and flux of laminar flows are of the form $\exp (-k)$. 


\section{ACKNOWLEDGEMENTS}

We thank Mohammad Anas for a valuable feedback on $\bar{k}_{d}$. Our numerical simulations were performed on Cray XC40 Shaheen II at KAUST supercomputing laboratory, Saudi Arabia and Chaos cluster of IIT Kanpur. This work was supported by the research grants PLANEX/PHY/2015239 from Indian Space Research Organisation India, INT/RUS/RSF/P-03 by the Department of Science and Technology India, and RSF-1641-02012 by Russian Science Foundation for the Indo-Russian project.

\section{REFERENCES}

${ }^{1}$ A. N. Kolmogorov, "The local structure of turbulence in incompressible viscous fluid for very large Reynolds numbers," Dokl Acad Nauk SSSR 30, 301-305 (1941).

${ }^{2} \mathrm{U}$. Frisch, Turbulence: The Legacy of A. N. Kolmogorov (Cambridge University Press, Cambridge, 1995).

${ }^{3}$ W. D. McComb, The physics of fluid turbulence, Oxford engineering science series (Clarendon Press, Oxford, 1990).

${ }^{4}$ P. A. Davidson, Turbulence: An Introduction for Scientists and Engineers (Oxford University Press, Oxford, 2004).

${ }^{5}$ T. Ishihara, T. Gotoh, and Y. Kaneda, "Study of high-Reynolds number isotropic turbulence by direct numerical simulation," Annu. Rev. Fluid Mech. 41, 165-180 (2009).

${ }^{6}$ D. C. Leslie, Developments in the theory of turbulence (Clarendon Press, Oxford, 1973).

${ }^{7}$ M. Lesieur, Turbulence in Fluids (Springer-Verlag, Dordrecht, 2008).

${ }^{8}$ S. B. Pope, Turbulent Flows (Cambridge University Press, Cambridge, 2000).

${ }^{9}$ Y.-H. Pao, "Structure of Turbulent Velocity and Scalar Fields at Large Wavenumbers," Phys. Fluids 8, 1063 (1965).

${ }^{10}$ D. O. Martínez, S. Chen, G. D. Doolen, R. H. Kraichnan, L.-P. Wang, and Y. Zhou, "Energy spectrum in the dissipation range of fluid turbulence," J. Plasma Phys. 57, 195201 (1997).

${ }^{11}$ S. G. Saddoughi and S. V. Veeravalli, "Local isotropy in turbulent boundary layers at high Reynolds number," J. Fluid Mech. 268, 333-372 (1994). 
${ }^{12}$ H. L. Grant, R. W. Stewart, and A. Moilliet, "Turbulence spectra from a tidal channel," J. Fluid Mech. 12, 241-268 (1962).

${ }^{13}$ T. Ishihara, Y. Kaneda, M. Yokokawa, K. Itakura, and A. Uno, "Energy Spectrum in the Near Dissipation Range of High Resolution Direct Numerical Simulation of Turbulence," J. Phys. Soc. Jpn. 74, 1464-1471 (2005).

${ }^{14}$ B. Lautrup, Physics of Continuous Matter, Second Edition: Exotic and Everyday Phenomena in the Macroscopic World, 2nd ed. (CRC Press, Boca Raton, FL, 2011).

${ }^{15}$ M. K. Verma, A. Kumar, and A. Pandey, "Phenomenology of buoyancy-driven turbulence: recent results," New J. Phys. 19, 025012 (2017).

${ }^{16}$ T. Gotoh and P. K. Yeung, "Passive scalar transport turbulence: a computational oerspective ," in Ten Chapters in Turbulence, edited by P. A. Davidson, Y. Kaneda, and K. R. Sreenivasan (Cambridge University Press, Cambridge, 2013) pp. 87-131.

${ }^{17}$ G. K. Batchelor, I. D. Howells, and A. A. Townsend, "Small-scale variation of convected quantities like temperature in turbulent fluid Part 2. The case of large conductivity," J. Fluid Mech. 5, 134-139 (1959).

${ }^{18}$ R. H. Kraichnan, "Small-scale structure of a scalar field convected by turbulence," Phys. Fluids 11, 945-953 (1968).

${ }^{19}$ M. F. Linkmann and A. N. Morozov, "Sudden Relaminarization and Lifetimes in Forced Isotropic Turbulence," Phys. Rev. Lett. 115, 134502 (2015).

${ }^{20}$ M. K. Verma, "Anisotropy in Quasi-Static Magnetohydrodynamic Turbulence," Rep. Prog. Phys. 80, 087001 (2017).

${ }^{21}$ J. A. Domaradzki and R. S. Rogallo, "Local Energy Transfer and Nonlocal Interactions in Homogeneous, Isotropic Turbulence," Phys. Fluids A 2, 414 (1990).

${ }^{22}$ Y. Zhou, "Degrees of locality of energy transfer in the inertial range," Phys. Fluids 5, 1092-1094 (1993).

${ }^{23}$ M. K. Verma, A. Ayyer, O. Debliquy, S. Kumar, and A. V. Chandra, "Local shell-toshell energy transfer via nonlocal interactions in fluid turbulence," Pramana-J. Phys. 65, 297-310 (2005).

${ }^{24}$ S. A. Orszag, "Analytical theories of turbulence," J. Fluid Mech. 41, 363-386 (1970).

${ }^{25}$ R. H. Kraichnan, "The structure of isotropic turbulence at very high Reynolds numbers," J. Fluid Mech. 5, 497-543 (1959). 
${ }^{26}$ M. K. Verma, A. G. Chatterjee, R. K. Yadav, S. Paul, M. Chandra, and R. Samtaney, "Benchmarking and scaling studies of pseudospectral code Tarang for turbulence simulations," Pramana-J. Phys. 81, 617-629 (2013).

${ }^{27}$ C. Canuto, M. Y. Hussaini, A. Quarteroni, and T. A. Zang, Spectral Methods in Fluid Dynamics (Springer-Verlag, Berlin Heidelberg, 1988).

${ }^{28}$ R. Stepanov, F. Plunian, M. Kessar, and G. Balarac, "Systematic bias in the calculation of spectral density from a three-dimensional spatial grid," Phys. Rev. E 90, 053309 (2014).

${ }^{29}$ G. Dar, M. K. Verma, and V. Eswaran, "Energy transfer in two-dimensional magnetohydrodynamic turbulence: formalism and numerical results," Physica D 157, 207-225 (2001).

${ }^{30}$ M. K. Verma, "Statistical theory of magnetohydrodynamic turbulence: recent results," Phys. Rep. 401, 229-380 (2004).

${ }^{31}$ K. S. Reddy and M. K. Verma, "Strong anisotropy in quasi-static magnetohydrodynamic turbulence for high interaction parameters," Phys. Fluids 26, 025109 (2014).

${ }^{32}$ K. R. Sreenivasan, "On the universality of the Kolmogorov constant," Phys. Fluids 7, 2778 (1995).

${ }^{33}$ P. K. Yeung and Y. Zhou, "Universality of the Kolmogorov constant in numerical simulations of turbulence," Phys. Rev. E 56, 1746-1752 (1997).

${ }^{34}$ T. Gotoh, D. Fukayama, and T. Nakano, "Velocity field statistics in homogeneous steady turbulence obtained using a high-resolution direct numerical simulation," Phys. Fluids 14, 1065-1081 (2002).

${ }^{35}$ M. Yokokawa, K. Itakura, A. Uno, T. Ishihara, and Y. Kaneda, "16.4-Tflops direct numerical simulation of turbulence by a Fourier spectral method on the Earth Simulator," in Proceeding Supercomputing 2002 (2002).

${ }^{36}$ P. D. Mininni, A. Alexakis, and A. Pouquet, "Nonlocal interactions in hydrodynamic turbulence at high Reynolds numbers: The slow emergence of scaling laws," Phys. Rev. E 77, 036306 (2008).

${ }^{37}$ D. A. Donzis and K. R. Sreenivasan, "The bottleneck effect and the Kolmogorov constant in isotropic turbulence," J. Fluid Mech. 657, 171-188 (2010).

${ }^{38}$ R. H. Kraichnan, "Inertial-range transfer in two-and three-dimensional turbulence," J. Fluid Mech. 47, 525-535 (1971). 
${ }^{39}$ V. Yakhot and S. A. Orszag, "Renormalization group analysis of turbulence. I. Basic theory," J. Sci. Comput. 1, 3-51 (1986).

${ }^{40}$ G. Falkovich, "Bottleneck phenomenon in developed turbulence," Phys. Fluids 6, 14111414 (1994).

${ }^{41}$ D. Lohse and A. Müller-Groeling, "Bottleneck effects in turbulence: Scaling phenomena in r versus p space," Phys. Rev. Lett. 74, 1747-1750 (1995).

${ }^{42}$ W. Dobler, N. E. L. Haugen, T. A. Yousef, and A. Brandenburg, "Bottleneck effect in three-dimensional turbulence simulations," Phys. Rev. E 68, 026304 (2003).

${ }^{43}$ M. K. Verma and D. A. Donzis, "Energy transfer and bottleneck effect in turbulence," J. Phys. A: Math. Theor. 40, 4401-4412 (2007).

${ }^{44}$ O. Debliquy, M. K. Verma, and D. Carati, "Energy fluxes and shell-to-shell transfers in three-dimensional decaying magnetohydrodynamic turbulence," Phys. Plasmas 12, 042309 (2005).

${ }^{45}$ G. Falkovich and A. Fouxon, "Anomalous scaling of a passive scalar in turbulence and in equilibrium," Phys. Rev. Lett. 94, 214502 (2005). 